Case Report:

\title{
Missed Forearm Artery Pseudoaneurysm Following a Direct Stab Wound
}

\author{
Ali Tabrizi ${ }^{1^{*}}$ iD, Hassan Taleb ${ }^{1}$, Mohammad Javad Shariyate ${ }^{2}$, Ahamdreza Afshar ${ }^{1}$ \\ 1. Clinical Research Development Unit of Imam Khomeini Hospital, Urmia University of Medical Sciences, Urmia, Iran \\ 2. Department of Orthopedics, Urmia University of Medical Sciences, Urmia, Iran.
}

$\begin{gathered}\text { Use your devic to scan } \\ \text { and read the article online }\end{gathered}$
. Journal of Research in Orthopedic Science. 2019; 6(3):27-30. http://dx.doi.org/10.32598/JROSJ.6.3.27
doi: $:$ http://dx.doi.org/10.32598/JROSJ.6.3.27

\section{(c) (i) (5)}

Article info:

Received: 31 Oct 2018

Revised: 22 Dec 2018

Accepted: 28 Jun 2018

Available Online: 01 Aug 2019

Keywords:

Stab wound;

Pseudoaneurysm;

Forearm

\begin{abstract}
A B S T RA C T
Introduction: Pseudoaneurysm, accompanied by penetrating trauma in the forearm, is rarely reported. Considering that clinical examination is not standard in these cases, clinical suspicion and application of suitable diagnostic modalities would be promising and helpful. Most of these patients were referred to the emergency units but were not correctly diagnosed.

Case Presentation: In this report, we present a 22-year-old patient with increasing pain because of a stab wound to forearm 10 days after the first referral to the emergency unit. A pseudoaneurysm was suspected in posterior interosseous of the radial artery because of the persistent pain and palpation of mass.

Conclusions: The results of bedside ultrasound in the emergency unit and CT angiography revealed pseudoaneurysm in posterior interosseous of the radial artery. The surgical treatment was the resection of the lesion and posterior interosseous ligature. All symptoms of the patient were resolved in a 2-months follow-up. Post-traumatic upper extremity pseudoaneurysm can show its signs and symptoms after a long time.
\end{abstract}

\section{Introduction}

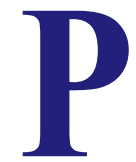

seudoaneurysm is rare in the radial artery, and only a few studies have been conducted in pseudoaneurysm as a result of penetrating trauma in the radial artery and, especially in the forearm [1]. The delayed manifestation of pseudoaneurysm in the arm and brachial artery has been reported [1-3]. Considering that clinical examinations are not reliable in these cases, the clinical suspicion and application of suitable diagnostic modalities would be helpful $[1,2]$. In this report, a delayed case of pseudoaneurysm is presented in the dorsolateral proximal of forearm region after a history of as penetrating trauma. Considering that in most cases its diagnosis is ignored, having strong clinical suspicion is very important.

\section{Case Presentation}

A 22-year-old male patient, suffering from pain, referred to the emergency unit of the university ten days

* Corresponding Author:

Ali Tabrizi, MD.

Address: Clinical Research Development Unit of Imam Khomeini Hospital, Urmia University of Medical Sciences, Urmia, Iran.

Phone: +98 (914) 3130829

E-mail:ali.tab.ms@gmail.com 


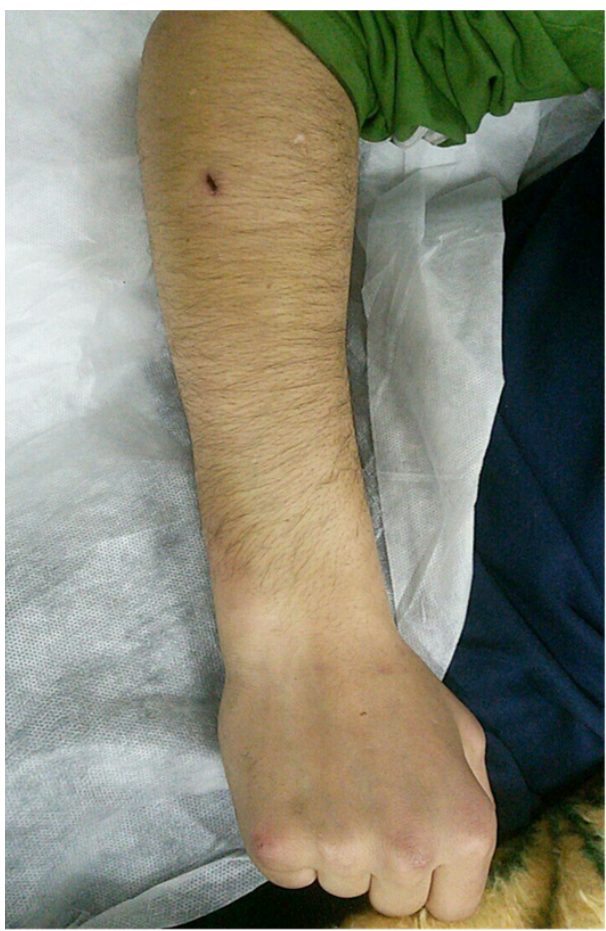

Figure 1. The scar of previously stab wound in the dorsolateral proximal of the forearm

Orthopedic Science

after a stab wound in the dorsolateral proximal of the forearm region. One day after the stab wound, he referred to the emergency unit and discharged after wound dressing and receiving oral antibiotic treatment. He had normal vascular, neural, and tendon functions at that time. After 10 days, the pain has increased in the fore- arm and intensified by wrist extension. The tenderness was in the place of the scar. Erythema was not observed. There were no symptoms of infection and secretions (Figure 1). There were no fever, chills, and local symptoms indicating deep infection and osteomyelitis. However, there was a $2 \mathrm{~cm}$ palpated mass in the scar position.

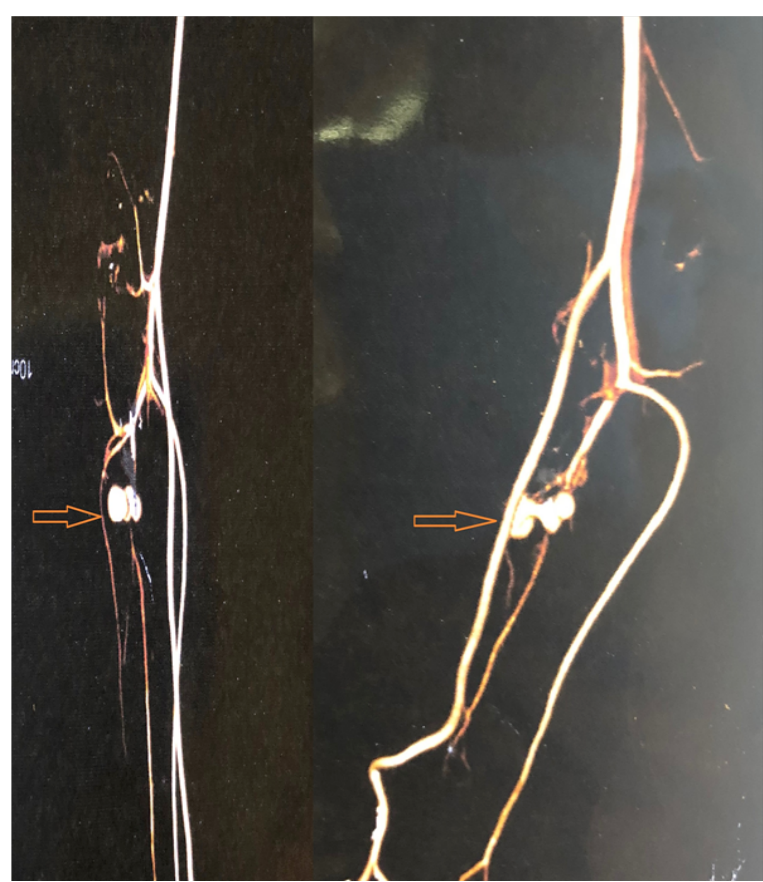

Orthopedic Science

Figure 2. Angiography of the forearm after 10 days of stab wound shows pseudoaneurysm in the posterior interosseous artery 


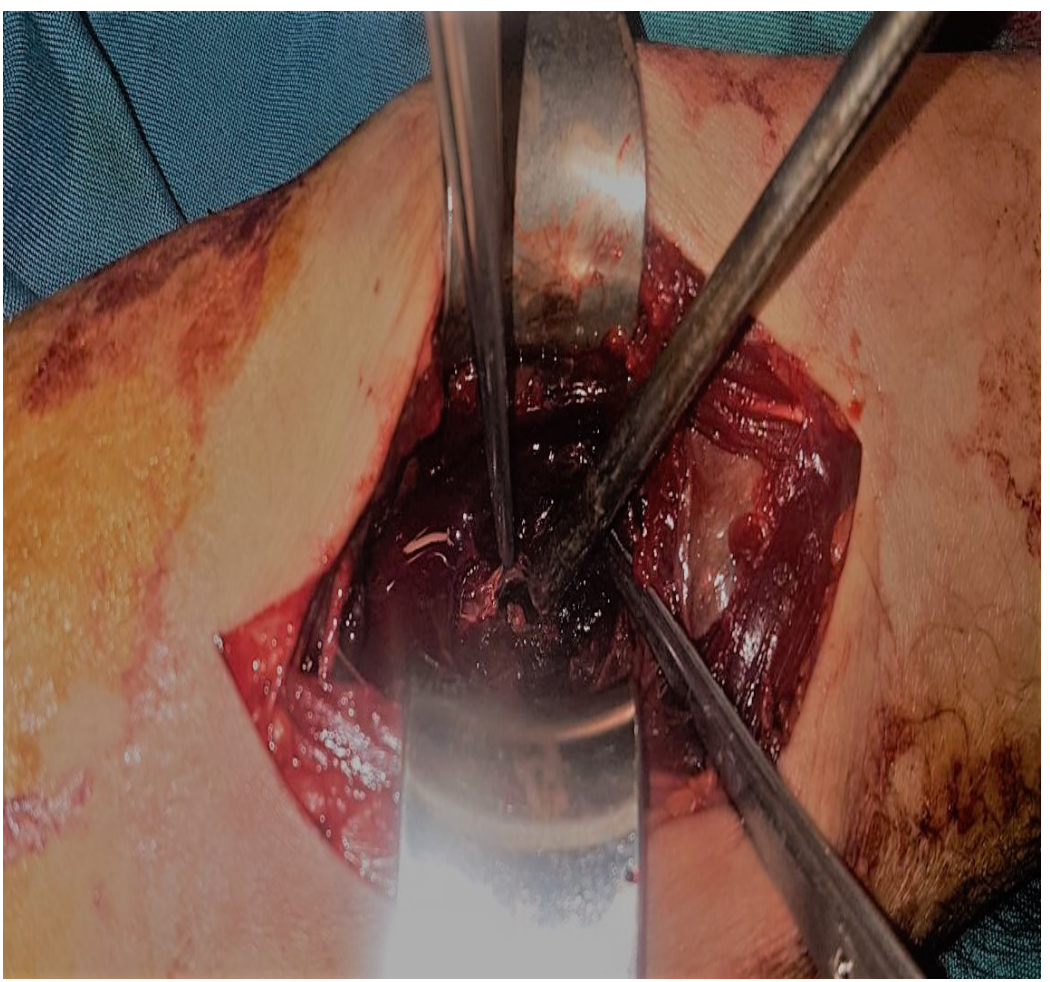

Figure 3. The exploration of the pseudoaneurysmal sac during operation

Orthopedic Science

The result of the Allen test was negative to roll out arterial insufficiency of the palm. Bedside ultrasound in the emergency unit detected a small cystic lesion $(1 \times 2 \mathrm{~cm})$, which was variable in size by probe pressure. Angiography results showed an aneurysm in the posterior interosseous branch of the radial artery (Figure 2).

Because of the involvement of posterior interosseous branch of the radial artery and unavailability of angiographic facilities for embolization, we resected the lesion by a longitudinal incision in the dorsal side of the proximal forearm and then ligated the posterior interosseous branch under general anesthesia and using tourniquet (Figure 3). When the tourniquet was off, the pulsating mass was visible at the site of exposure. We resected lesion then ligated proximal part of the lesion. Two weeks after the operation, all the patient's symptoms disappeared. After 2-month follow-up and physiotherapy, the patient regained his activity level and ability in the upper limb.

\section{Conclusions}

Hematoma caused by a stab wound may result in the false aneurysm, called pseudoaneurysm [1]. The local enlargement of the blood vessel usually occurs in arteries as a result of traumatic perforation of the walls of the vessel. Blood leakage from arteries' wall in surrounding tissue causes a hematoma, which occurs after a while and not clearing in early stages. Thus, there is a high probability of being missed in the emergency units [1, 2]. Also, the clinical manifestations of pseudoaneurysm might start from some days to some months after trauma and range from continuous discomfort since the trauma, increased pain, and swelling [3].

Notably, in the main arteries, pseudoaneurysm is risky and can be raptured [3]. Pseudoaneurysm followed by penetrating trauma has been rarely reported in upper limb compared to lower limb. Based on the present documents, no reasonable evidence is available for estimating the required time for the formation of these pseudoaneurysms [3]. In two reports, the reported time was more than one month after penetrating trauma and laceration in the upper limb $[2,3]$. Komorwska et al. reported more clinical evidence in 16 cases of pseudoaneurysm caused by infected catheter in the distal radial artery, but less evidence in the cases caused by penetrating trauma [4].

In the report of Forde et al., the delayed manifestation of pseudoaneurysm in the brachial artery was followed by penetrating trauma in a 23 -year-old individual [2]. Three months after the penetrating trauma incidence, the authors found swelling and tenderness in antecubital of the elbow; the treatment with surgical resection led to symptoms relief [2]. In a report by Fokou et al., large 
pseudoaneurysm is introduced in the axillary artery followed by a stab wound after two months with pain and neural symptoms in brachial plexus [5]. The operation was carried out through interposition saphenous vein [5].

However, vascular embolization treatment can be successful in unavailable and sensitive regions. Solomon stated that pseudoaneurysm resulted from penetrating trauma in the temporal artery was successfully treated by embolization [6]. Depending on aneurysm sac and created mass, it might be tender with pain or swelling. If you touch the mass, it can be palpated, but there is no thrill $[4,5]$. The clinical examination is often unreliable, and the strong clinical suspension is helpful. The application of suitable diagnostic modalities would be promising and helpful for prompt diagnosis.

\section{Ethical Considerations}

\section{Compliance with ethical guidelines}

The case report was confirmed by the Ethics Committee of Urmia University of Medical Sciences.

\section{Funding}

This research did not receive any specific grant from funding agencies in the public, commercial, or not-forprofit sectors.

\section{Authors' contributions}

All authors contributed in preparing this article.

\section{Conflict of interest}

The authors declared no conflict of interests. pseudoaneurysms using percutaneous thrombin injection. J Hand Surgery. 2004; 29:936-42. [DOI:10.1016/j. jhsa.2004.05.009] [PMID]

[5] Fokou M, Eyenga VC, Mefire AC, Guifo ML, Pagbe JJ, Sandmann W. Giant pseudoaneurysm of the left axillary artery following a stab wound. Cardiovasc J Afr. 2012; 23(9):e4-6. [DOI:10.5830/CVJA-2012-045] [PMID]

[6] Solomons TL. Pseudo aneurysm in an artery following a stab wound. S Afr Radiographer. 2009; 47(2):23-4.

\section{References}

[1] Pero T, Herrick J. Pseudoaneurysm of the radial artery diagnosed by bedside ultrasound. West J Emerg Med. 2009; 10(2):89-91. [PMID] [PMCID]

[2] Forde JC, Conneely JB, Aly S. Delayed presentation of a traumatic brachial artery pseudoaneurysm. Ulus Travma Acil Cerrahi Derg. 2009; 15(5):515-7. [PMID]

[3] Yetkin U, Gurbuz A. Post-traumatic pseudoaneurysm of the brachial artery and its surgical treatment. Tex Heart Inst J. 2003; 30(4):293-7. [PMID] [PMCID]

[4] Komorowska-Timek E, Teruya TH, Abou-Zamzam Jr AM, Papa D, Ballard JL. Treatment of radial and ulnar artery 